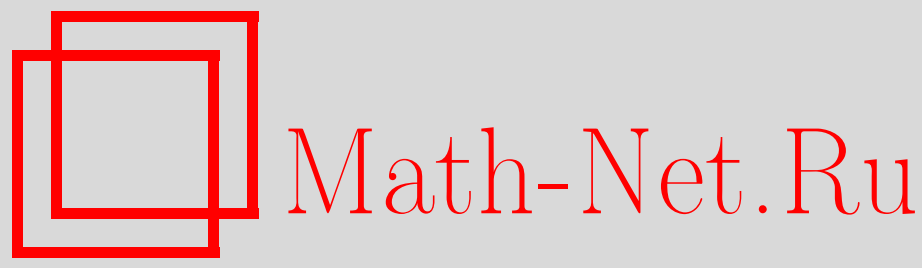

Е. Е. Дьяконова, Ветвящиеся процессы в марковской случайной среде, Дискрет. матем., 2014, том 26, выпуск $3,10-29$

DOI: https://doi.org/10.4213/dm1287

Использование Общероссийского математического портала Math-Net.Ru подразумевает, что вы прочитали и согласны с пользовательским соглашением http://www . mathnet.ru/rus/agreement

Параметры загрузки:

IP : 18.208 .226 .222

26 апреля 2023 г., 02:29:47

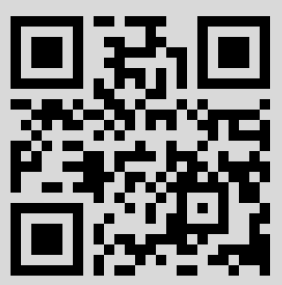




\section{Ветвящиеся процессы в марковской случайной среде}

(c) 2014 г. .. Е. Дьяконова*

Рассматривается ветвящийся процесс в случайной марковской среде, обладающий свойством критичности. Доказаны условная функциональная предельная теорема для числа частиц в процессе и условный принцип инвариантности. Найдены асимптотики хвостов распределения максимума числа частиц в процессе и общего числа частиц в процессе.

Работа выполнена при финансовой поддержке программы РАН "Динамические системы и теория управления".

Ключевые слова: ветвящиеся процессы, функциональная предельная теорема, марковская случайная среда.

\section{1. Введение и основные результаты}

Впервые ветвящийся процесс в случайной среде был рассмотрен в основополагающей работе Смита и Вилкинсона [1] для случая среды, порожденной последовательностью независимых одинаково распределенных случайных величин. Затем Атрейя и Карлин [2] проанализировали свойства ветвящихся процессов, находящихся под влиянием среды более общего вида. С тех пор было опубликовано большое количество работ, посвященных изучению ветвящихся процессов в случайной среде (см., например, [6]-[22]). При этом в основном рассматривалась ситуация среды, порожденной последовательностью независимых одинаково распределенных случайных величин. Именно для этой ситуации асимптотика вероятности невырождения критического ветвящегосяся процесса при условии конечности второго момента $\mathbf{E} X^{2}<\infty$ приращения $X$ сопровождающего случайного блуждания была исследована в статьях [6] и [12]. В работах [7]-[9] также в предположении $\mathbf{E} X^{2}<\infty$ были доказаны теоремы, описывающие поведение числа частиц в критических процессах, в частности, найдены асимптотики хвостов распределений максимального числа частиц в процессе и общего числа частиц за время жизни процесса. В последующих работах [10], [14]-[16], [20] была получена асимптотика вероятности невырождения и изучено поведение числа частиц в процессе для гораздо более широкого класса критических ветвящихся процессов с одним типом частиц, эволюционирующих в случайной среде, порожденной последовательностью независимых одинаково распределенных случайных величин. Для представителей этого класса не только конечность второго момента $\mathbf{E} X^{2}$ не обязательна, но даже и существование математического ожидания у случайной величины $X$ не является необходимым требованием.

*Место работы: Математический институт им. В.А. Стеклова РАН, e-mail: elena@mi.ras.ru 
Для случайных сред более общего вида вероятность невырождения критических и докритических ветвящихся процессов с одним типом частиц в стационарной случайной среде изучалась в [22]. Асимптотика вероятности невырождения критического ветвящегося процесса с одним типом частиц для случая марковской среды с конечным множеством состояний была получена в статье [21]. В [18] была найдена асимптотика вероятности невырождения критического ветвящегося процесса с $p \geqslant 1$ типами частиц, эволюционирующего в марковской случайной среде со счетным множеством состояний, а затем в статье [19] исследовано предельное поведение числа частиц в этом процессе в момент времени $n$ при $n \rightarrow \infty$.

В настоящей работе для ветвящегося процесса в случайной марковской среде со счетным множеством состояний, обладающего свойством критичности, получен ряд предельных теорем, характеризующих поведение числа частиц в процессе. В частности, мы обобщаем некоторые результаты работ [18] и [19], а также распространяем некоторые результаты статей [9] и [10] на случай марковской случайной среды со счетным множеством состояний.

Рассматриваемая нами модель ветвящегося процесса в случайной среде может быть описана следующим образом. Пусть $\mathbf{N}:=\{1,2, \ldots\}, \mathbf{N}_{0}:=\mathbf{N} \cup\{0\}$ и $\mathbf{e}=$ $\left\{e_{n}, n \in \mathbf{N}_{0}\right\}$ - неприводимая апериодичная положительно возвратная цепь Маркова с множеством состояний $\mathbf{N}_{0}$. С каждым значением $i \in \mathbf{N}_{0}$ свяжем вероятностную производящую функцию $f^{(i)}(s), s \in[0,1]$. Последовательность случайных неотрицательных целочисленных случайных величин $\left\{Z_{n}, n \in \mathbf{N}_{0}\right\}$ называется ветвящимся процессом Гальтона-Ватсона в марковской случайной среде е, если $Z_{0}$ не зависит от е и для всех $n \in \mathbf{N}_{0}, z \in \mathbf{N}_{0}$ и $i_{0}, i_{1}, \ldots \in \mathbf{N}_{0}$

$$
\mathcal{L}\left(Z_{n+1} \mid Z_{n}=z, \mathbf{e}=\left(i_{0}, i_{1}, \ldots\right)\right)=\mathcal{L}\left(\sum_{j=1}^{z} \xi_{n}^{(j)}\right)
$$

где случайные целочисленные неотрицательные величины $\xi_{n}^{(1)}, \xi_{n}^{(2)}, \ldots, \xi_{n}^{(z)}$ независимы в совокупности и распределены согласно вероятностной производящей функции $f^{\left(i_{n}\right)}(s)$.

Соотношение (1) задает ветвящийся процесс Гальтона-Ватсона в случайной марковской среде, в котором величина $Z_{n}$ - число частиц в $n$-м поколении, причем частицы в этом процессе эволюционируют следующим образом. Если $e_{n}=i \in \mathbf{N}_{0}$, то все $Z_{n}$ частиц $n$-го поколения производят потомков согласно вероятностному закону, заданному производящей функцией $f^{(i)}(s)$, независимо друг от друга и от предыстории процесса. Таким образом, размножение частиц из $n$-го поколения происходит согласно производящей функции

$$
f_{n}(s):=f^{\left(e_{n}\right)}(s) .
$$

Будем называть средой случайную последовательность $\left(e_{0}, e_{1}, e_{2}, \ldots\right)$. Предполагается, что процесс начался с одной частицы, т. е. $Z_{0}=1$.

Для любой вероятностной меры $\lambda$ на $\mathbf{N}_{0}$ символы $\mathbf{E}^{(\lambda)}$ и $\mathbf{P}^{(\lambda)}$ будут обозначать математическое ожидание и, соответственно, вероятность, относящиеся к рассматриваемому ветвящемуся процессу $\left\{Z_{n}\right\}$ в случайной среде, имеющей начальное распределение $\lambda$. Если при этом начальная мера $\lambda$ целиком сосредоточена в точке $i \in \mathbf{N}_{0}$, то будем использовать символы $\mathbf{E}^{(i)}$ и $\mathbf{P}^{(i)}$ соответственно. Там, где это не приводит к недоразумениям, будем опускать верхние индексы в этих обозначениях 
писать просто $\mathbf{E}$ и $\mathbf{P}$. Символы $\mathbb{P}, \mathbb{E}$ и $\mathbb{D}$, играющие существенную роль в последующих рассуждениях, используются для обозначения условной вероятности, условного математического ожидания и условной дисперсии при фиксированной среде $\left(e_{0}, e_{1}, e_{2}, \ldots\right)$.

Положим для $0 \leqslant k<n-1$

$$
f_{k, n}(s):=f_{k}\left(f_{k+1}\left(\ldots\left(f_{n-1}(s)\right) \ldots\right)\right), f_{n-1, n}(s):=f_{n-1}(s) .
$$

Далее, если не оговорено противное, будем считать, что начальное состояние $e_{0}=0$. Обозначим $\nu \geqslant 1$ момент первого возвращения марковской цепи $\left\{e_{n}\right\}$ в состояние 0 . Пусть

$$
f_{0, \nu}(s):=f_{0}\left(f_{1}\left(\ldots\left(f_{\nu-1}(s)\right) \ldots\right)\right)
$$

- итерации, построенные по последовательности производящих функций $f_{0}(s), f_{1}(s), \ldots, f_{\nu-1}(s)$. Всюду в дальнейшем будем предполагать, что для всех $i \in \mathbf{N}_{0}$ у производящей функции $f^{(i)}(s)$ существуют конечные производные первого и второго порядков в точке 1, причем производные первого порядка положительны. Положим

$$
X:=\ln f_{0, \nu}^{\prime}(1), \eta:=\frac{f_{0, \nu}^{\prime \prime}(1)}{\left(f_{0, \nu}^{\prime}(1)\right)^{2}}
$$

Будем предполагать, что ветвящийся процесс $\left\{Z_{n}, n \in \mathbf{N}_{0}\right\}$ в случайной среде $\mathbf{e}$ удовлетворяет следующим условиям.

Условие А1. Найдется такое $\delta>0$, что

$$
\mathbf{E} \nu^{2+\delta}<\infty
$$

Условие А2. Выполняются соотношения

$$
\begin{gathered}
\mathbf{E} X=0,0<\sigma^{2}:=\mathbf{E} X^{2}<\infty, \\
\mathbf{E} \ln ^{q}(\max \{\eta, 1\})<+\infty,
\end{gathered}
$$

для некоторого $q>2$.

Таким образом, условие А2 постулирует критичность рассматриваемого процесса. Пусть

$$
X_{i}:=\ln f_{i-1}^{\prime}(1), i \in \mathbf{N}
$$

Положим

$$
S_{0}:=0, S_{n}:=X_{1}+\ldots+X_{n}, n \in \mathbf{N} \text {. }
$$

Введем случайные величины

$$
\begin{aligned}
\eta_{i}:=\frac{f_{i}^{\prime \prime}(1)}{\left(f_{i}^{\prime}(1)\right)^{2}}, i & \in \mathbf{N}_{0}, \quad \mu:=\sum_{i=0}^{\nu-1}\left(\eta_{i}+1\right) e^{-S_{i}}, \\
\chi & :=\max _{i=0, \ldots, \nu-1}\left|S_{i}\right| .
\end{aligned}
$$

Положим при $x>0$

$$
g(x):=\sum_{k=0}^{\infty} \mathbf{P}(\mu \geqslant x, \nu>k), \quad \phi(x):=\sum_{k=0}^{\infty} \mathbf{P}(\chi \geqslant x, \nu>k) .
$$


Условие А3. Существует $\beta>1 / 2$ такое, что

$$
\lim _{x \rightarrow \infty} x^{\beta} g(x)=0
$$

Условие А4. Выполняется соотношение

$$
\lim _{x \rightarrow \infty} x^{3} \phi(x)=0
$$

Положим для $t \in[0,1]$

$$
Y_{n t}:=\frac{Z_{n t}}{\exp \left\{S_{n t}\right\}}, Y_{n t}:=\left\{Y_{n}, t \in[0,1]\right\} .
$$

Здесь и далее в аналогичных ситуациях мы будем понимать $n t$ как целую часть числа $n t$, чтобы не загромождать формулы.

Теорема 1. Пусть выполнены условия $A 1-A 3$. Тогда

$$
\left\{Y_{n t}, t \in(0,1] \mid Z_{n}>0\right\} \stackrel{\mathcal{D}}{\rightarrow}\left\{G_{t}, t \in(0,1]\right\}
$$

где предельный процесс $\left\{G_{t}\right\}$ является процессом с п.н. постоянными положительными траекториями, т.е.

$$
\mathbf{P}\left(G_{t}=G \text { для всех } t \in(0,1]\right)=1,
$$

причем

$$
\mathbf{P}(0<G<\infty)=1
$$

Сходимость в (8) означает слабую сходимость в пространстве $D[u, 1]$ с топологией Скорохода [3] при любом и $\in(0,1)$.

Пусть $\left\{W^{+}(t), t \in[0,1]\right\}$ - броуновская извилина [3]. Мы будем понимать под $W^{+}(t)$ при $t>1$ стандартное броуновское движение, начинающееся из $W^{+}(1)$ и не зависящее от траектории процесса $\left\{W^{+}(t)\right\}$ до момента времени 1 , и использовать обозначение $W^{+}=\left\{W^{+}(t), t \in[0, \infty]\right\}$. Пусть

$$
\tau:=\inf \left\{t>0: W^{+}(t)=0\right\} .
$$

Отметим, что $\tau>1$ так как $W^{+}(t)>0$ при $t \in(0,1]$. Положим $V:=\mathbf{E} \nu$.

Следующее утверждение является принципом инвариантности для ветвящегося процесса в марковской среде.

Теорема 2. Пусть выполнены условия $A 1-A 4$. Тогда

$$
\left\{\frac{\ln Z_{n t}}{\sigma \sqrt{n}}, t \in[0,1] \mid Z_{n}>0\right\} \stackrel{\mathcal{D}}{\rightarrow}\left\{\frac{W^{+}(t)}{\sqrt{V}}, t \in[0,1]\right\} .
$$

Сходимость в (10) означает слабую сходимость в пространстве $D[0,1]$ с топологией Скорохода. 


\section{2. Вспомогательные результаты}

Пусть

$$
\nu(0):=0, \nu(n+1):=\min \left\{k>\nu(n): e_{k}=0\right\}, n \in \mathbf{N}_{0},
$$

- последовательность моментов попадания марковской цепи $\left\{e_{n}\right\}$ в начальное состояние $e_{0}=0$. Очевидно, что последовательность $\{\nu(n)\}$ образует процесс восстановления, приращения которого $\nu(n+1)-\nu(n), n \in \mathbf{N}_{0}$, распределены так же, как и случайная величина $\nu:=\nu(1)$, причем ее среднее $V:=\mathbf{E} \nu \geqslant 1$. Введем вложенный ветвящийся процесс (см. [18], [19]):

$$
Z_{n}^{*}:=Z_{\nu(n)}, n \in \mathbf{N}_{0} .
$$

Ясно, что процесс $\left\{Z_{n}^{*}, n \in \mathbf{N}_{0}\right\}$ является ветвящимся процессом Гальтона-Ватсона, функционирующим в случайной среде с независимыми одинаково распределенными компонентами. При этом размножение частиц $n$-го поколения процесса $\left\{Z_{n}^{*}\right\}$ описывается производящей функцией

$$
f_{* n}(s):=f_{\nu(n)}\left(f_{\nu(n)+1}\left(\ldots\left(f_{\nu(n+1)-1}(s)\right) \ldots\right)\right) .
$$

Положим

$$
X_{k}^{*}:=\ln f_{* k-1}(s), \eta_{k+1}^{*}:=\frac{f_{* k}^{\prime \prime}(1)}{\left(f_{* k}^{\prime}(1)\right)^{2}}, k \in \mathbf{N}_{0} .
$$

Очевидно, что $X_{j}^{*} \stackrel{d}{=} X_{1}^{*}:=\ln f_{0, \nu-1}^{\prime}(1)$. Введем для процесса $\left\{Z_{n}^{*}\right\}$ сопровождающее его случайное блуждание

$$
S_{0}^{*}=0, S_{n}^{*}=X_{1}^{*}+\ldots+X_{n}^{*}, n \in \mathbf{N} .
$$

Заметим, что $S_{n}^{*}=S_{\nu(n)}$. Определим при $n \in \mathbf{N}_{0}$ случайные величины $r=r(n)$ и $m=m(n)$ соотношениями

$$
\begin{aligned}
r(n) & :=\max \{k: \nu(k) \leqslant n\}, \\
m(n) & :=\max \left\{k \leqslant n: e_{k}=0\right\} .
\end{aligned}
$$

Отметим, что $S_{r(n)}^{*}=S_{m(n)}, Z_{r(n)}^{*}=Z_{m(n)}$. Ясно, что

$$
S_{n}=S_{r(n)}^{*}+\ln f_{m(n), n}^{\prime}(1),
$$

где $\ln f_{n, n}^{\prime}(s):=0$. Для $t \in[0,1]$ положим

$$
Y_{n t}^{*}:=\frac{Z_{n t}^{*}}{\exp \left\{S_{n t}^{*}\right\}}, Y_{n}^{*}:=\left\{Y_{n t}^{*}, t \in[0,1]\right\} .
$$

Лемма 1. Пусть выполнено условие А2. Тогда при $n \rightarrow \infty$

$$
\mathbf{P}\left(Z_{n}^{*}>0\right) \sim c_{0} n^{-1 / 2},
$$

где $c_{0}>0$, а также

$$
\left\{Y_{n t}^{*}, t \in(0,1] \mid Z_{n}^{*}>0\right\} \stackrel{\mathcal{D}}{\rightarrow}\left\{G_{t}, t \in(0,1]\right\},
$$


где предельный прочесс $\left\{G_{t}\right\}$ является процессом с п.н. постоянными положительными траекториями, т.е.

$$
\mathbf{P}\left(G_{t}=G \text { для всех } t \in(0,1]\right)=1,
$$

причем

$$
\mathbf{P}(0<G<\infty)=1 \text {. }
$$

Кроме того, при $n \rightarrow \infty$

$$
\left\{\frac{S_{n t}^{*}}{\sigma \sqrt{n}}, t \in[0,1] \mid Z_{n}^{*}>0\right\} \stackrel{\mathcal{D}}{\rightarrow}\left\{W^{+}(t), t \in[0,1]\right\},
$$

где $\sigma-и з(2), W^{+}(\cdot)-$ броуновская извилина.

Для доказательства достаточно заметить, что из выполнения условия А2 следует, что случайная величина $X_{1}^{*}$ принадлежит области притяжения нормального распределения и процесс $\left\{Z_{n}^{*}\right\}$ удовлетворяет условиям теорем $1.3,1.5$ и следствия 1.2 из [10], согласно которым справедливы соотношения (14) - (16).

Нам также понадобится следующее утверждение, являющееся комбинацией следствия 1 из [18] и леммы 4 из [19].

Лемма 2. Пусть выполнены условия $A 1$ и A2. Тогда при $n \rightarrow \infty$

$$
\mathbf{P}\left(Z_{n}>0\right) \sim c_{1} n^{-1 / 2},
$$

где $c_{1}=c_{0} \sqrt{V}$, а $c_{0}-$ из леммы 1 .

Лемма 3. Пусть выполнень условия $A 1$ и $A 2$. Тогда при $n \rightarrow \infty$

$$
\left\{\frac{S_{r(n t)}^{*}}{\sigma \sqrt{n}}, t \in[0,1] \mid Z_{n}>0\right\} \stackrel{\mathcal{D}}{\rightarrow}\left\{\frac{W^{+}(t)}{\sqrt{V}}, t \in[0,1]\right\} .
$$

Доказательство. Зафиксируем произвольное $\varepsilon_{0} \in(0,1 / 2)$, произвольные $k \in \mathbf{N}$, $x_{i} \geqslant 0$ и $0<t_{1}<t_{2}<\ldots<t_{k} \leqslant 1$. Пусть

$$
n^{-}:=n\left(1-\varepsilon_{0}\right) / V, n^{+}:=n\left(1+\varepsilon_{0}\right) / V \text {. }
$$

Для $t>0$ введем интервал

$$
U_{n t}=U_{n t}\left(\varepsilon_{0}\right):=\left[n^{-} t, n^{+} t\right], U_{n}:=U_{n 1} .
$$

Из формул (26), (36) и (37) работы [18] следует, что для любого фиксированного $u \in(0,1)$ при $n \rightarrow \infty$

$$
n^{\delta+1} \mathbf{P}\left(r(n t) \notin U_{n t}\right) \rightarrow 0
$$

равномерно по $t \in[u, 1]$, где величина $\delta$ та же, что и в условии А1. Откуда

$$
\begin{aligned}
& \sqrt{n} \mathbf{P}\left(\frac{S_{r\left(n t_{i}\right)}^{*}}{\sigma \sqrt{n}}>x_{i}, i=1, \ldots, k, Z_{n}>0\right) \\
\sim & \sqrt{n} \mathbf{P}\left(\frac{S_{r\left(n t_{i}\right)}^{*}}{\sigma \sqrt{n}}>x_{i}, r\left(n t_{i}\right) \in U_{n t_{i}}, i=1, \ldots, k, r(n) \in U_{n}, Z_{n}>0\right) .
\end{aligned}
$$


Учитывая, что

$$
\left\{Z_{r(n)}^{*}>0\right\} \supseteq\left\{Z_{n}>0\right\} \supseteq\left\{Z_{r(n)+1}^{*}>0\right\},
$$

несложно видеть, что в силу произвольности $\varepsilon_{0}$ и непрерывности почти всех траекторий процесса $\left\{W^{+}(t)\right\}$ из $(14),(16),(17),(22),(23)$ следует сходимость конечномерных распределений последовательности процессов $\left\{\frac{S_{r(n t)}^{*}}{\sigma \sqrt{n}}, t \in[0,1] \mid Z_{n}>0\right\} \mathrm{K}$ конечномерным распределениям процесса $\left\{\frac{W^{+}(t)}{\sqrt{V}}\right\}$.

Для $\delta>0$ и функции $F=F(t) \in D[a, b], 0 \leqslant a<b<\infty$, введем модуль непрерывности

$$
w_{F}(\delta, a, b):=\sup _{\substack{|x-s|<\delta, x, s \in[a, b]}}|F(x)-F(s)| .
$$

Положим для $t \in[0,1]$

$$
\begin{gathered}
\mathcal{S}_{n}^{*}(t):=\frac{S_{r(n t)}^{*}}{\sigma \sqrt{n}}, \mathcal{S}_{n}^{* *}(t):=\frac{S_{n t}^{*}}{\sigma \sqrt{n}}, \\
\mathcal{S}_{n}^{*}:=\left\{\mathcal{S}_{n}^{*}(t), t \in[0,1]\right\}, \mathcal{S}_{n}^{* *}:=\left\{\mathcal{S}_{n}^{* *}(t), t \in[0,1]\right\} .
\end{gathered}
$$

Докажем, что для произвольного $\varepsilon>0$

$$
\lim _{\delta \downarrow 0} \varlimsup_{n \rightarrow \infty} \mathbf{P}\left(w_{\mathcal{S}_{n}^{*}}(\delta, 0,1)>\varepsilon \mid Z_{n}>0\right)=0 .
$$

Покажем, что

$$
\lim _{\delta \downarrow 0} \overline{\lim _{n \rightarrow \infty}} \sqrt{n} \mathbf{P}\left(w_{\mathcal{S}_{n}^{*}}(\delta, 0,1)>\varepsilon, Z_{r(n)}^{*}>0\right)=0 .
$$

Из леммы 1 следует, что для любого $\varepsilon^{\prime}>0$ существует такое $\delta^{\prime} \in(0,1 / V)$, что при

$$
\varlimsup_{n \rightarrow \infty} \sqrt{n} \mathbf{P}\left(w_{\mathcal{S}_{n}^{* *}}\left(\delta^{\prime}, 0,1\right)>\varepsilon \sqrt{V / 2}, Z_{n}^{*}>0\right) \leqslant \frac{\varepsilon^{\prime}}{\sqrt{2 V}}
$$

а значит, существует такое $n_{0}>0$, что при $n>n_{0}$

$$
\mathbf{P}\left(w_{\mathcal{S}_{n}^{* *}}\left(\delta^{\prime}, 0,1\right)>\varepsilon \sqrt{V / 2}, Z_{n}^{*}>0\right) \leqslant \frac{\varepsilon^{\prime}}{\sqrt{2 V n}} .
$$

Зададим значение величины $\varepsilon_{0}$ в $(19)$

$$
\varepsilon_{0}:=\frac{\delta^{\prime} V}{4} .
$$

Положим $m:=\left[1 / \varepsilon_{0}\right]+1$, где $[x]-$ целая часть числа $x$. Пусть

$$
y_{i}=\frac{i}{m}, i=0, \ldots, m
$$

а интервалы $U_{n y_{i}}=U_{n y_{i}}\left(\varepsilon_{0}\right), i=1, \ldots, m,-$ те же, что и в $(20)$. В силу $(21)$ для $\delta \in(0,1)$

$$
\begin{aligned}
& \varlimsup_{n \rightarrow \infty} \sqrt{n} \mathbf{P}\left(w_{\mathcal{S}_{n}^{*}}(\delta, 0,1)>\varepsilon, Z_{r(n)}^{*}>0\right) \\
& =\varlimsup_{n \rightarrow \infty} \sqrt{n} \mathbf{P}\left(w_{\mathcal{S}_{n}^{*}}(\delta, 0,1)>\varepsilon, Z_{r(n)}^{*}>0, r\left(n y_{i}\right) \in U_{n y_{i}}, i=1, \ldots, m\right) .
\end{aligned}
$$


Для $s \in[0,1]$ положим

$$
y^{-}(s):=\max _{i}\left\{y_{i}: y_{i} \leqslant s\right\}, y^{+}(s):=\min _{i}\left\{y_{i}: y_{i} \geqslant s\right\}
$$

Так как $r\left(n_{1}\right) \leqslant r\left(n_{2}\right)$ при $n_{1} \leqslant n_{2}$, то при $r\left(n y_{i}\right) \in U_{n y_{i}}, i=1, \ldots, m$, для таких $x, s$, что $0 \leqslant s \leqslant x \leqslant 1, x-s \leqslant \delta_{0}:=1 / m$,

$$
\begin{aligned}
r(n x)-r(n s) & \leqslant r\left(n y^{+}(x)\right)-r\left(n y^{-}(s)\right) \\
& \leqslant y^{+}(x) \frac{n\left(1+\varepsilon_{0}\right)}{V}-y^{-}(s) \frac{n\left(1-\varepsilon_{0}\right)}{V} \\
& \leqslant \frac{n}{V}\left(\frac{2}{m}+2 \varepsilon_{0}\right) \leqslant n \delta^{\prime} .
\end{aligned}
$$

Поскольку $\varepsilon_{0} \leqslant 1 / 2$, и, значит, при $r(n) \in U_{n}$

$$
\frac{n}{2 V} \leqslant r(n) \leqslant \frac{2 n}{V}
$$

из (28) следует, что

$$
\begin{aligned}
& \mathbf{P}\left(w_{\mathcal{S}_{n}^{*}}\left(\delta_{0}, 0,1\right)>\varepsilon, Z_{r(n)}^{*}>0, r\left(n y_{i}\right) \in U_{n y_{i}}, i=1, \ldots, m\right) \\
& \leqslant \mathbf{P}\left(\frac{w_{\mathcal{S}_{n}^{*}}\left(\delta_{0}, 0,1\right) \sqrt{n}}{\sqrt{r(n)}}>\varepsilon \sqrt{V / 2}, Z_{r(n)}^{*}>0, r\left(n y_{i}\right) \in U_{n y_{i}}, i=1, \ldots, m\right) \\
& \quad \leqslant \sup _{k \in U_{n}} \mathbf{P}\left(w_{\mathcal{S}_{k}^{* *}}\left(\delta^{\prime}, 0,1\right)>\varepsilon \sqrt{V / 2}, Z_{k}^{*}>0\right) .
\end{aligned}
$$

Из (26) и (29) вытекает, что при $n>2 V n_{0}$

$$
\sup _{k \in U_{n}} \mathbf{P}\left(w_{\mathcal{S}_{k}^{* *}}\left(\delta^{\prime}, 0,1\right)>\varepsilon \sqrt{V / 2}, Z_{k}^{*}>0\right) \leqslant \frac{\varepsilon^{\prime}}{\sqrt{n}} .
$$

Из (27), (30) и (31) получаем, что при $n>2 V n_{0}$

$$
\varlimsup_{n \rightarrow \infty} \sqrt{n} \mathbf{P}\left(w_{\mathcal{S}_{n}^{*}}\left(\delta_{0}, 0,1\right)>\varepsilon, Z_{r(n)}^{*}>0\right) \leqslant \varepsilon^{\prime},
$$

откуда вытекает равенство (25), которое в силу (17), (21) и (23) влечет справедливость (24). Из сходимости конечномерных распределений и формулы (24) следует (см. [3] § 15) соотношение (18).

Лемма доказана.

Лемма 4. При выполнении условий $A 1-A 4$

$$
\left\{\frac{S_{n t}}{\sigma \sqrt{n}}, t \in[0,1] \mid Z_{n}>0\right\} \stackrel{\mathcal{D}}{\rightarrow}\left\{\frac{W^{+}(t)}{\sqrt{V}}, t \in[0,1]\right\} .
$$

Доказательство. Положим для $t \in[0,1]$

$$
\begin{gathered}
\Delta_{n, t}:=S_{n t}-S_{r(n t)}^{*} \\
\mathcal{S}_{n}(t):=\frac{S_{n t}}{\sigma \sqrt{n}}, \mathcal{R}_{n}(t):=\frac{\Delta_{n, t}}{\sigma \sqrt{n}}
\end{gathered}
$$




$$
\mathcal{S}_{n}:=\left\{\mathcal{S}_{n}(t), t \in[0,1]\right\}, \mathcal{R}_{n}:=\left\{\mathcal{R}_{n}(t), t \in[0,1]\right\}
$$

Очевидно, что

$$
\mathcal{S}_{n}=\mathcal{S}_{n}^{*}+\mathcal{R}_{n}
$$

Поэтому в силу лемм 2 и 3 для доказательства сходимости (32) достаточно показать, что для произвольного $\varepsilon>0$ при $n \rightarrow \infty$

$$
\sqrt{n} \mathbf{P}\left(\sup _{t \in[0,1]}\left|\mathcal{R}_{n}(t)\right|>\varepsilon, Z_{n}>0\right) \rightarrow 0 .
$$

Ясно, что

$$
\mathbf{P}\left(\sup _{t \in[0,1]}\left|\mathcal{R}_{n}(t)\right|>\varepsilon, Z_{n}>0\right) \leqslant \mathbf{P}\left(\sup _{t \in[0,1]}\left|\Delta_{n, t}\right|>\varepsilon \sigma \sqrt{n}\right) .
$$

Положим для $i=0,1, \ldots$

$$
\chi_{i}:=\max _{j=\nu(i), \ldots, \nu(i+1)-1}\left|S_{j}-S_{\nu(i)}^{*}\right|, \nu_{i}:=\nu(i+1)-\nu(i) .
$$

Несложно видеть, что $\left(\chi_{0}, \nu_{0}\right),\left(\chi_{1}, \nu_{1}\right), \ldots$ образуют последовательность независимых одинаково распределенных случайных двумерных векторов, причем $\left(\chi_{i}, \nu_{i}\right) \stackrel{d}{=}$ $(\chi, \nu)$, где $\chi$ из $(6)$.

Следовательно, при $x>0$

$$
\mathbf{P}\left(\sup _{t \in[0,1]}\left|\Delta_{n, t}\right|>x\right) \leqslant \sum_{i=0}^{r(n)} \sum_{k=0}^{\infty} \mathbf{P}\left(\chi_{i}>x, \nu_{i}>k\right) \leqslant n \phi(x) .
$$

Из соотношений (35) и (36) в силу условия $A 4$ следует (32). Лемма доказана.

\section{3. Доказательства теорем}

Доказательство теоремы 1. Зафиксируем $u \in(0,1)$ и $t \in[u, 1]$. Покажем сначала, что для $x \geqslant 0$ при $n \rightarrow \infty$

$$
\mathbf{P}\left(Y_{n t}>x \mid Z_{n}>0\right) \rightarrow \mathbf{P}\left(G_{t}>x\right),
$$

где случайная величина $G_{t}$ та же, что и в (15). Для этого воспользуемся схемой доказательства теоремы 1 в [19], в которой установлено соотношение (37) для случая $t=1$. Здесь мы приведем соответствующие аргументы только для полноты изложения.

Для $0 \leqslant n_{1}<n_{2}$ обозначим через $\xi^{(k)}\left(n_{1}, n_{2}\right)$ численность потомства в момент времени $n_{2}$ частицы, которая жила в $n_{1}$-м поколении и была в нём $k$-й, $k=1, \ldots, Z_{n_{1}}$. Положим $\xi^{(k)}\left(n_{2}, n_{2}\right)=1$. Рассмотрим величину

$$
\mathbf{P}\left(Y_{n t}>x, Z_{n}>0\right)=\mathbf{P}\left(Y_{r(n t)}^{*}+\varkappa_{r(n t)}>x, Z_{n}>0\right)
$$

где

$$
\varkappa_{r(n t)}:=Y_{n t}-Y_{r(n t)}^{*}=e^{-S_{r(n t)}^{*}} \sum_{k=1}^{Z_{r(n t)}^{*}}\left(\frac{\xi^{(k)}(m(n t), n t)}{e^{S_{n t}-S_{m(n t)}}}-1\right) .
$$


Зафиксируем произвольное $\varepsilon_{0} \in(0,1 / 2)$. Ясно, что

$$
\begin{aligned}
\mathbf{P}\left(Y_{n t}>x, Z_{n}>0\right)= & \mathbf{P}\left(Y_{n t}>x, Z_{n}>0, r(n t) \in U_{n t}, r(n) \in U_{n}\right) \\
& +\mathbf{P}\left(Y_{n t}>x, Z_{n}>0, r(n t) \notin U_{n t}, r(n) \in U_{n}\right) \\
& +\mathbf{P}\left(Y_{n t}>x, Z_{n}>0, r(n t) \notin U_{n t}, r(n) \notin U_{n}\right) \\
& +\mathbf{P}\left(Y_{n t}>x, Z_{n}>0, r(n t) \in U_{n t}, r(n) \notin U_{n}\right),
\end{aligned}
$$

где интервалы $U_{n t}, U_{n}$ те же, что и в (20). Из (17) и (21) следует, что для доказательства (37) достаточно рассмотреть первый член в правой части равенства (40). Для $i=0,1$ положим

$$
\mathcal{A}_{i}:=\mathbf{P}\left(Y_{n t}>x, Z_{r(n)+i}^{*}>0, r(n t) \in U_{n t}, r(n) \in U_{n}\right) .
$$

Несложно видеть, что

$$
\mathcal{A}_{0} \geqslant \mathbf{P}\left(Y_{n t}>x, Z_{n}>0, r(n t) \in U_{n t}, r(n) \in U_{n}\right) \geqslant \mathcal{A}_{1} .
$$

Покажем, что

$$
\lim _{n \rightarrow \infty} \frac{\sqrt{n}}{c_{0} \sqrt{V}} \mathcal{A}_{i}=\mathbf{P}\left(G_{t}>x\right), i=0,1,
$$

где $c_{0}$ - из (14), $G_{t}$ - из (15). Мы рассмотрим лишь случай $i=0$, поскольку для $i=1$ доказательство аналогично. Согласно (38)

$$
\mathcal{A}_{0}=\mathbf{P}\left(Y_{r(n t)}^{*}+\varkappa_{r(n t)}>x, Z_{r(n)}^{*}>0, r(n t) \in U_{n t}, r(n) \in U_{n}\right) .
$$

Из леммы 1 и соотношения (21) следует, что

$$
\lim _{n \rightarrow \infty} \frac{\sqrt{n}}{c_{0} \sqrt{V}} \mathbf{P}\left(Y_{r(n t)}^{*}>x, Z_{r(n)}^{*}>0, r(n t) \in U_{n t}, r(n) \in U_{n}\right)=\mathbf{P}\left(G_{t}>x\right) .
$$

Покажем, что для произвольного $\varepsilon>0$ при $n \rightarrow \infty$

$$
\sqrt{n} \mathbf{P}\left(\left|\varkappa_{r(n t)}\right|>\varepsilon, Z_{r(n)}^{*}>0, r(n t) \in U_{n t}, r(n) \in U_{n}\right) \rightarrow 0
$$

равномерно по $t \in[u, 1]$. Пусть для целых неотрицательных $k$

$$
\tau^{*}(k):=\min \left\{j \leqslant k: S_{j}^{*}=\min \left(S_{0}^{*}, \ldots, S_{k}^{*}\right)\right\}
$$

- самая левая точка, в которой случайное блуждание $\left\{S_{j}^{*}\right\}$ на интервале $[0, k]$ достигает минимума. Для $0 \leqslant l \leqslant k$ обозначим

$$
L_{l, k}^{*}:=\min _{0 \leqslant j \leqslant k-l}\left(S_{l+j}^{*}-S_{l}^{*}\right), L_{k}^{*}:=L_{0, k}^{*},
$$

т.е. $L_{k}^{*}$ - минимум случайного блуждания $S_{0}^{*}, \ldots, S_{k}^{*}$ на интервале $[0, k]$. Очевидно, что

$$
\left\{\tau^{*}(k)=l\right\}=\left\{\tau^{*}(l)=l\right\} \cap\left\{L_{l, k}^{*} \geqslant 0\right\} .
$$

Запишем величину $\beta$ из условия А 3 в виде

$$
\beta=\frac{1 / 2+\varepsilon_{2}}{1-\varepsilon_{1}},
$$


где $\varepsilon_{1} \in(0,1)$ и $\varepsilon_{2}>0$. Для $M=\left[n^{\varepsilon_{1} / 4}\right]$ (здесь и далее $[x]$ обозначает целую часть числа $x)$ рассмотрим представление

$$
\begin{gathered}
\quad \mathbf{P}\left(\left|\varkappa_{r(n t)}\right|>\varepsilon, Z_{r(n)}^{*}>0, r(n t) \in U_{n t}, r(n) \in U_{n}\right) \\
=\quad \mathbf{P}\left(\left|\varkappa_{r(n t)}\right|>\varepsilon, Z_{r(n)}^{*}>0, \tau^{*}(r(n t))>M, r(n t) \in U_{n t}, r(n) \in U_{n}\right) \\
+\mathbf{P}\left(\left|\varkappa_{r(n t)}\right|>\varepsilon, Z_{r(n)}^{*}>0, \tau^{*}(r(n t)) \leqslant M, r(n t) \in U_{n t}, r(n) \in U_{n}\right) \\
=: \mathcal{H}(n, t, M)+\mathcal{C}(n, t, M) .
\end{gathered}
$$

Ясно, что

$$
\begin{aligned}
\mathcal{H}(n, t, M) \leqslant & \mathbf{P}\left(Z_{r(n)}^{*}>0, \tau^{*}(r(n t))>M, r(n t) \in U_{n t}\right) \\
\leqslant & \mathbf{P}\left(Z_{n^{-} t}^{*}>0, \tau^{*}\left(n^{+} t\right)>M, r(n t) \in U_{n t}\right) \\
= & \mathbf{P}\left(Z_{n^{-} t}^{*}>0, Z_{n^{+} t}^{*}>0, \tau^{*}\left(n^{+} t\right)>M, r(n t) \in U_{n t}\right) \\
& +\mathbf{P}\left(Z_{n^{-} t}^{*}>0, Z_{n^{+} t}^{*}=0, \tau^{*}\left(n^{+} t\right)>M, r(n t) \in U_{n t}\right) \\
\leqslant & \mathcal{H}_{1}(n, t, M)+\mathcal{H}_{2}(n, t),
\end{aligned}
$$

где

$$
\begin{gathered}
\mathcal{H}_{1}(n, t, M):=\mathbf{P}\left(Z_{n^{+} t}^{*}>0, \tau^{*}\left(n^{+} t\right)>M\right), \\
\mathcal{H}_{2}(n, t):=\mathbf{P}\left(Z_{n^{-} t}^{*}>0, Z_{n^{+} t}^{*}=0\right) .
\end{gathered}
$$

Из формулы (4.3) работы [10] следует, что при $k \rightarrow \infty$

$$
\varlimsup_{n \rightarrow \infty} \sqrt{n} \mathbf{P}\left(Z_{n^{+} t}^{*}>0, \tau^{*}\left(n^{+} t\right)>k\right) \rightarrow 0
$$

равномерно по $t \in[u, 1]$. Следовательно, существует такая положительная функция $\gamma(n)$, что при всех $t \in[u, 1]$

$$
\sqrt{n} \mathcal{H}_{1}(n, t, M) \leqslant \gamma(n) \rightarrow 0, n \rightarrow \infty .
$$

Поскольку

$$
\mathcal{H}_{2}(n, t)=\mathbf{P}\left(Z_{n^{-} t}^{*}>0\right)-\mathbf{P}\left(Z_{n^{+} t}^{*}>0\right)
$$

и $\varepsilon_{0} \in(0,1 / 2)$, то, применяя (14), получаем, что

$$
\begin{aligned}
\sqrt{n} \mathcal{H}_{2}(n, t) & \leqslant \frac{c_{2}}{4 \sqrt{t}}\left(\frac{1}{\sqrt{1-\varepsilon_{0}}}-\frac{1}{\sqrt{1+\varepsilon_{0}}}\right) \\
& \leqslant \frac{c_{2}}{4 \sqrt{u}}\left(\frac{1+\varepsilon_{0}}{1-\varepsilon_{0}}-1\right) \leqslant \frac{c_{2}}{\sqrt{u}} \varepsilon_{0}
\end{aligned}
$$

для некоторого $c_{2}>0$. Из (49), (52) и (53), учитывая произвольность $\varepsilon_{0}$, получаем, что при $n \rightarrow \infty$

$$
\sqrt{n} \mathcal{H}(n, t, M) \rightarrow 0
$$

равномерно по $t \in[u, 1]$.

Рассмотрим теперь величину $\mathcal{C}(n, t, M)$. Ясно, что

$$
\mathcal{C}(n, t, M) \leqslant \mathbf{P}\left(\left|\varkappa_{r(n t)}\right|>\varepsilon, \tau^{*}(r(n t)) \leqslant M, r(n t) \in U_{n t}\right)=: \mathcal{C}_{0}(n, t, M) .
$$


Для $N=\left[n^{\varepsilon_{1} / 2}\right]$ запишем представление

$$
\mathcal{C}_{0}(n, t, M)=\mathcal{C}_{1}(n, t, M, N)+\mathcal{C}_{2}(n, t, M, N)
$$

где

$$
\begin{gathered}
\mathcal{C}_{1}(n, t, M, N):=\sum_{l=0}^{M} \mathbf{P}\left(\left|\varkappa_{r(n t)}\right|>\varepsilon, e^{-S_{l}^{*}}>N, \tau^{*}(r(n t))=l, r(n t) \in U_{n t}\right), \\
\mathcal{C}_{2}(n, t, M, N):=\sum_{l=0}^{M} \mathbf{P}\left(\left|\varkappa_{r(n t)}\right|>\varepsilon, e^{-S_{l}^{*}} \leqslant N, \tau^{*}(r(n t))=l, r(n t) \in U_{n t}\right) .
\end{gathered}
$$

\section{Положим}

$$
f_{* l, k}(s):=f_{* l}\left(f_{* l+1}\left(\ldots\left(f_{* k-1}(s)\right) \ldots\right)\right), 0 \leqslant l \leqslant k-2, f_{* k-1, k}(s):=f_{* k-1}(s),
$$

где $f_{* k}(s)$ - из (11). Ясно, что $f_{* k}^{\prime}(1)=e^{S_{\nu(k+1)}-S_{\nu(k)}}, f_{* 0, k}^{\prime}(1)=e^{S_{k}^{*}}$. Заметим, что (см. (46))

$$
\begin{aligned}
& \mathcal{C}_{1}(n, t, M, N) \leqslant \sum_{l=0}^{M} \mathbf{P}\left(Z_{r(n t)}^{*}>0, e^{-S_{l}^{*}}>N, \tau^{*}(r(n t))=l, r(n t) \in U_{n t}\right) \\
& =\mathbf{E}\left[\sum_{l=0}^{M} \mathbb{P}\left(Z_{r(n t)}^{*}>0, e^{-S_{l}^{*}}>N, \tau^{*}(r(n t))=l, r(n t) \in U_{n t}\right)\right] \\
& \leqslant \mathbf{E}\left[\sum_{l=0}^{M}\left(1-f_{* 0, r(n t)}(0)\right) I\left\{e^{-S_{l}^{*}}>N, \tau^{*}(r(n t))=l, r(n t) \in U_{n t}\right\}\right] \\
& \leqslant \mathbf{E}\left[\sum_{l=0}^{M} e^{S_{l}^{*}}\left(1-f_{* l, r(n t)}(0)\right) I\left\{e^{-S_{l}^{*}}>N, \tau^{*}(r(n t))=l, r(n t) \in U_{n t}\right\}\right] \\
& \leqslant \frac{1}{N} \mathbf{E}\left[\sum_{l=0}^{M}\left(1-f_{* l, r(n t)}(0)\right) I\left\{\tau^{*}(r(n t))=l, r(n t) \in U_{n t}\right\}\right] \\
& \leqslant \frac{1}{N} \sum_{l=0}^{M} \mathbf{P}\left(\tau^{*}(l)=l\right) \mathbf{E}\left[\left(1-f_{* l, r(n t)}(0)\right) ; L_{l, r(n t)}^{*} \geqslant 0, r(n t) \in U_{n t}\right],
\end{aligned}
$$

где $I\{\mathcal{B}\}$ - индикатор события $\mathcal{B}$, а величина $L_{l, k}^{*}$ та же, что и в $(45)$. Из формул (1.7) и (4.8) в [10] следует, что при $n \rightarrow \infty$

$$
\begin{aligned}
& \mathbf{P}\left(Z_{n}^{*}>0, L_{n}^{*} \geqslant 0\right)=\mathbf{E}\left[\mathbb{P}\left(Z_{n}^{*}>0, L_{n}^{*} \geqslant 0\right)\right] \\
= & \mathbf{E}\left[\left(1-f_{* 0, n}(0)\right) I\left\{L_{n}^{*} \geqslant 0\right\}\right] \sim c_{3} \mathbf{P}\left(Z_{n}^{*}>0\right),
\end{aligned}
$$

где $c_{3}>0$, а величина $L_{n}^{*}$ та же, что и в (45). Так как при $l \in\{0,1, \ldots, M\}$

$$
\begin{gathered}
\mathbf{E}\left[1-f_{* l, r(n t)}(0) ; L_{l, r(n t)}^{*} \geqslant 0, r(n t) \in U_{n t}\right] \\
\leqslant \mathbf{E}\left[1-f_{* l, n^{-} t}(0) ; L_{l, n^{-} t}^{*} \geqslant 0\right]
\end{gathered}
$$


то объединяя (58) - (60) и используя (14), получаем, что для некоторого $c_{4}>0$

$$
\mathcal{C}_{1}(n, t, M, N) \leqslant \frac{M+1}{N} c_{4}\left(1-\varepsilon_{0}\right)^{-1 / 2} u^{-1 / 2} n^{-1 / 2}=o\left(n^{-1 / 2}\right), n \rightarrow \infty .
$$

Обозначим через

$$
m^{+}(n t):=\min \left\{k \geqslant n t: e_{k}=0\right\}
$$

следующий после $m(n t)$ момент попадания цепи $\left\{e_{n}\right\}$ в состояние 0. Пусть

$$
\Xi(n t):=\sum_{k=m(n t)}^{n t}\left(\frac{f_{k}^{\prime \prime}(1)}{\left(f_{k}^{\prime}(1)\right)^{2}}+1\right) e^{-S_{k}}, \Xi^{(1)}(n t):=\sum_{k=m(n t)}^{m^{+}(n t)}\left(\frac{f_{k}^{\prime \prime}(1)}{\left(f_{k}^{\prime}(1)\right)^{2}}+1\right) e^{-S_{k}}
$$

Положим $x_{n, t}:=(n t)^{1-\varepsilon_{1}}$, где $\varepsilon_{1}-$ из $(47)$. Представим $\mathcal{C}_{2}(n, M, N)$ из (57) в виде суммы

$$
\mathcal{C}_{2}(n, t, M, N)=\mathcal{C}_{2}^{(1)}(n, t, M, N)+\mathcal{C}_{2}^{(2)}(n, t, M, N)
$$

где

$$
\begin{aligned}
& \mathcal{C}_{2}^{(1)}(n, t, M, N):= \\
& \sum_{l=0}^{M} \mathbf{P}\left(\left|\varkappa_{r(n t)}\right|>\varepsilon, e^{-S_{l}^{*}} \leqslant N, \Xi(n t)>x_{n, t}, \tau^{*}(r(n t))=l, r(n t) \in U_{n t}\right), \\
& \mathcal{C}_{2}^{(2)}(n, t, M, N):= \\
& \sum_{l=0}^{M} \mathbf{P}\left(\left|\varkappa_{r(n t)}\right|>\varepsilon, e^{-S_{l}^{*}} \leqslant N, \Xi(n t) \leqslant x_{n, t}, \tau^{*}(r(n t))=l, r(n t) \in U_{n t}\right) .
\end{aligned}
$$

Поскольку $\Xi^{(1)}(n t) \geqslant \Xi(n t)$, то

$$
\begin{aligned}
\mathcal{C}_{2}^{(1)}(n, t, M, N) & \leqslant \mathbf{P}\left(\Xi(n t)>x_{n, t}, r(n t) \in U_{n t}\right) \\
& \leqslant \mathbf{P}\left(\Xi^{(1)}(n t)>x_{n, t}, r(n t) \in U_{n t}\right)=: \mathcal{C}_{2}^{(1,1)}(n, t) .
\end{aligned}
$$

Пусть

$$
\begin{gathered}
\nu_{n t}^{-}:=n t-\nu(r(n t)), A(y):=\mathbf{P}(\nu \leqslant y), \\
H(k):=\sum_{l=1}^{\infty} A^{l *}(k), h(k+1):=H(k+1)-H(k), k \in \mathbf{N}_{0},
\end{gathered}
$$

где $A^{l *}(y)-l$-кратная свертка функции распределения $A(y)$ с собой.

Так как $\nu(n) \geqslant n$ и $\nu(r(n)) \leqslant n$, то

$$
\begin{aligned}
\mathcal{C}_{2}^{(1,1)}(n, t) & \leqslant \mathbf{P}\left(\Xi^{(1)}(n t)>x_{n, t}, n^{-} t \leqslant r(n t)\right) \\
& \leqslant \mathbf{P}\left(\Xi^{(1)}(n t)>x_{n, t}, n^{-} t \leqslant \nu(r(n t)) \leqslant n t\right) \\
& =\sum_{k=0}^{n t-n^{-} t} \mathbf{P}\left(\Xi^{(1)}(n t)>x_{n, t}, \nu_{n t}^{-}=k\right) \\
& \leqslant \sum_{k=0}^{n t-n^{-} t} h(n t-k) \mathbf{P}\left(\mu>x_{n, t}, \nu>k\right)=: \mathcal{C}_{2}^{(1,2)}(n, t),
\end{aligned}
$$


где величина $n^{-}$та же, что и в (19). По теореме восстановления (см. [13], гл. 9, §2) при достаточно больших $n$ имеем для $t \in[u, 1]$

$$
\mathcal{C}_{2}^{(1,2)}(n, t,) \leqslant \frac{2}{V} \sum_{k=0}^{\infty} \mathbf{P}\left(\mu>x_{n, t}, \nu>k\right) \leqslant \frac{2}{V} \sum_{k=0}^{\infty} \mathbf{P}\left(\mu>x_{n, u}, \nu>k\right) .
$$

Из (64), (65) и (66) в силу условия А3 следует, что при $n \rightarrow \infty$

$$
\sqrt{n} \mathcal{C}_{2}^{(1)}(n, t, M, N) \rightarrow 0
$$

равномерно по $t \in[u, 1]$.

Для оценки величины $\mathcal{C}_{2}^{(2)}(n, t, M, N)$ воспользуемся равенством

$$
\begin{aligned}
& \mathcal{C}_{2}^{(2)}(n, t, M, N) \\
= & \mathbf{E}\left[\sum_{l=0}^{M} \mathbb{P}\left(\left|\varkappa_{r(n t)}\right|>\varepsilon, e^{-S_{l}^{*}} \leqslant N, \Xi(n t) \leqslant x_{n, t}, \tau^{*}(r(n t))=l, r(n t) \in U_{n t}\right)\right] .
\end{aligned}
$$

Положим для $n_{1} \leqslant n_{2}$

$$
\rho^{(k)}\left(n_{1}, n_{2}\right):=\frac{\xi^{(k)}\left(n_{1}, n_{2}\right)}{\exp \left\{S_{n_{2}}-S_{n_{1}}\right\}}-1, k=1, \ldots, Z\left(n_{1}\right),
$$

где $\xi^{(k)}\left(n_{1}, n_{2}\right)$ были определены перед (38). Ясно, что при фиксированной среде случайные величины $\xi^{(k)}\left(n_{1}, n_{2}\right), k=1, \ldots, Z\left(n_{1}\right)$, независимы и одинаково распределены. Так как $\mathbb{E}\left[Z_{m(n t)}\right]=e^{S_{r(n t)}^{*}}$ и

$$
\varkappa_{r(n t)}=\frac{1}{e^{S_{r(n t)}^{*}}} \sum_{k=1}^{Z_{m(n t)}} \rho^{(k)}(m(n t), n t),
$$

то в силу неравенства Чебышева

$$
\mathbb{P}\left(\left|\varkappa_{r(n t)}\right|>\varepsilon\right) \leqslant \frac{1}{\varepsilon^{2} e^{2 S_{r(n t)}^{*}}} e^{S_{r(n t)}^{*}} \mathbb{D}\left[\rho^{(1)}(m(n t), n t)\right] .
$$

Несложно видеть, что $\mathbb{E}\left[\xi^{(k)}\left(n_{1}, n_{2}\right)\right]=e^{S_{n_{2}}-S_{n_{1}}}$ и

$$
\mathbb{E}\left[\xi^{(k)}\left(n_{1}, n_{2}\right)\left(\xi^{(k)}\left(n_{1}, n_{2}\right)-1\right)\right]=e^{2\left(S_{n_{2}}-S_{n_{1}}\right)} \sum_{k=n_{1}}^{n_{2}-1} \frac{f_{k}^{\prime \prime}(1)}{\left(f_{k}^{\prime}(1)\right)^{2}} e^{-\left(S_{k}-S_{n_{1}}\right)},
$$

то есть

$$
\mathbb{D}\left[\rho^{(1)}(m(n t), n t)\right] \leqslant \Xi(n t),
$$

где $\Xi(n t)$ - из соотношения (62). Объединяя (68) - (71), получаем

$$
\begin{aligned}
& \mathcal{C}_{2}^{(2)}(n, t, M, N)
\end{aligned}
$$

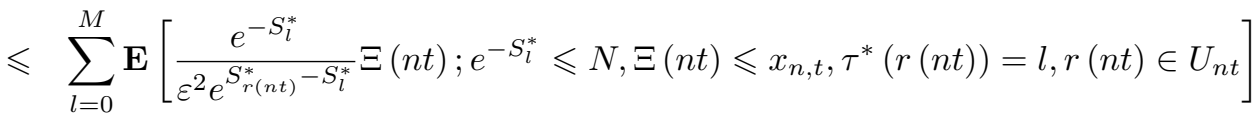

$$
\begin{aligned}
& \leqslant \frac{N x_{n, t}}{\varepsilon^{2}} \sum_{l=0}^{M} \mathbf{E}\left[e^{-\left(S_{r(n t)}^{*}-S_{l}^{*}\right)} ; \tau^{*}(r(n t))=l, r(n t) \in U_{n t}\right] \text {. }
\end{aligned}
$$


В силу утверждения 2.1 работы [11]

$$
\mathbf{E}\left[e^{-S_{k}^{*}}, L_{k}^{*} \geqslant 0\right] \sim c_{5} k^{-3 / 2}, k \rightarrow \infty,
$$

где $c_{5}>0$. Из $(72)$ и (73) следует, что при $n \rightarrow \infty$

$$
\mathcal{C}_{2}^{(2)}(n, t, M, N) \leqslant \frac{c_{6} N(M+1) x_{n, t}}{\varepsilon^{2}} n^{-3 / 2} \leqslant \frac{c_{6} N(M+1)}{\varepsilon^{2}} n^{1-\varepsilon_{1}-3 / 2}=o\left(n^{-1 / 2}\right),
$$

где $c_{6}>0$. Из соотношений $(55),(56),(67)$ и $(74)$, учитывая произвольность $\varepsilon_{0}$, получаем, что при $n \rightarrow \infty$

$$
\sqrt{n} \mathcal{C}(n, t, M) \rightarrow 0
$$

равномерно по $t \in[u, 1]$. Соотношения (48), (54) и (75) влекут выполнение (44).

Теперь, объединяя (42) - (44), получаем (41) для случая $i=0$. Из (40), (41) в силу произвольности $\varepsilon_{0}$ следует $(37)$.

Рассмотрим теперь конечномерные распределения процесса $\left\{Y_{n t} \mid Z_{n}>0\right\}$. Зафиксируем $k \in \mathbf{N}, x_{i} \geqslant 0$ и $t_{i} \in[u, 1], i=1, \ldots, k$, причем $u \leqslant t_{1}<t_{2}<\ldots<t_{k} \leqslant 1$. Из соотношения (21) следует, что

$$
\begin{aligned}
& \sqrt{n} \mathbf{P}\left(Y_{n t_{i}}>x_{i}, i=1, \ldots, k, Z_{n}>0\right) \\
& \quad \sim \sqrt{n} \mathbf{P}\left(Y_{n t_{i}}>x_{i}, r\left(n t_{i}\right) \in U_{n t_{i}}, i=1, \ldots, k, r(n) \in U_{n}, Z_{n}>0\right) .
\end{aligned}
$$

Так как $Y_{n t_{i}}=Y_{r\left(n t_{i}\right)}^{*}+\varkappa_{r\left(n t_{i}\right)}$ и для произвольного $\varepsilon>0, i=1, \ldots, k$, в силу $(44)$

$\mathbf{P}\left(\exists j \in\{1, \ldots, k\}:\left|\varkappa_{r\left(n t_{j}\right)}\right|>\varepsilon, r\left(n t_{i}\right) \in U_{n t_{i}}, i=1, \ldots, k, r(n) \in U_{n}, Z_{n}>0\right)$

$$
\leqslant \sum_{i=1}^{k} \mathbf{P}\left(\left|\varkappa_{r\left(n t_{i}\right)}\right|>\varepsilon, r\left(n t_{i}\right) \in U_{n t_{i}}, r(n) \in U_{n}, Z_{n}>0\right)=o\left(n^{-1 / 2}\right),
$$

то из $(17),(76),(77)$ и леммы 1 следует, что конечномерные распределения последовательности процессов $\left\{Y_{n t} \mid Z_{n}>0\right\}$ сходятся при $n \rightarrow \infty$ к конечномерным распределениям процесса $\left\{G_{t}\right\}$.

Для завершения доказательства осталось убедиться, что при любом $\varepsilon>0$

$$
\lim _{\delta \downarrow 0} \varlimsup_{n \rightarrow \infty} \mathbf{P}\left(w_{Y_{n}}(\delta, u, 1)>\varepsilon \mid Z_{n}>0\right)=0 .
$$

Из леммы 1 и соотношения (21), применяя рассуждения, аналогичные приведенным при доказательстве соотношения (25), заключаем, что

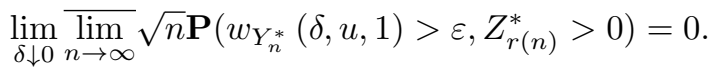

Откуда, учитывая соотношения (14), (17), (21), (39) и (44), несложно получить (78). Теорема доказана.

Доказательство теоремы 2. Если $Z_{n}>0$, то положим при $t \in[0,1]$

$$
\mathcal{X}_{n, t}:=\frac{\ln Y_{n t}}{\sigma \sqrt{n}}, \mathcal{Z}_{n, t}:=\frac{\ln Z_{n t}}{\sigma \sqrt{n}}, \mathcal{X}_{n}=\left\{\mathcal{X}_{n, t}, t \in[0,1]\right\}, \mathcal{Z}_{n}=\left\{\mathcal{Z}_{n, t}, t \in[0,1]\right\}
$$

Из теоремы 1 следует, что при $n \rightarrow \infty$

$$
\left\{\mathcal{X}_{n, t}, t \in(0,1] \mid Z_{n}>0\right\} \stackrel{\mathcal{D}}{\rightarrow} 0 .
$$


Откуда, применяя лемму 4, получаем

$$
\left\{\mathcal{Z}_{n, t}, t \in(0,1] \mid Z_{n}>0\right\} \stackrel{\mathcal{D}}{\rightarrow}\left\{\frac{W^{+}(t)}{\sqrt{V}}, t \in(0,1]\right\}
$$

Таким образом, для завершения доказательства осталось показать, что для любого $\varepsilon>0$

$$
\lim _{u \downarrow 0} \lim _{\delta \downarrow 0} \varlimsup_{n \rightarrow \infty} \mathbf{P}\left(w_{\mathcal{Z}_{n}}(\delta, 0, u)>\varepsilon \mid Z_{n}>0\right)=0
$$

Легко видеть, что

$$
w_{\mathcal{Z}_{n}}(\delta, 0, u) \leqslant \sup _{t \in[0, u]} \frac{\ln Z_{n t}}{\sigma \sqrt{n}} \leqslant \sup _{t \in[0, u]} \frac{S_{n t}}{\sigma \sqrt{n}}+\sup _{t \in[0, u]} \frac{\ln Y_{n t}}{\sigma \sqrt{n}} .
$$

Заметим, что для $\varepsilon>0$ в силу леммы 4 и непрерывности траекторий броуновской извилины

$$
\lim _{u \downarrow 0} \lim _{n \rightarrow \infty} \mathbf{P}\left(\sup _{t \in[0, u]} \frac{S_{n t}}{\sigma \sqrt{n}}>\varepsilon \mid Z_{n}>0\right)=\lim _{u \downarrow 0} \mathbf{P}\left(\sup _{t \in[0, u]} W^{+}(t)>\varepsilon\right)=0 .
$$

Так как $\mathbb{E}\left[Z_{n} e^{-S_{n}}\right]=1$ и при фиксированной случайной среде последовательность $\left\{Z_{n} e^{-S_{n}}, n \in \mathbf{N}\right\}$ образует мартингал, то в силу неравенства Дуба получаем, что для $\varepsilon>0$ и $u \in(0,1)$

$$
\mathbb{P}\left(\sup _{t \in[0, u]} \frac{\ln Y_{n t}}{\sigma \sqrt{n}}>\varepsilon, Z_{n}>0\right) \leqslant \mathbb{P}\left(\sup _{k \leqslant n} \frac{Z_{k}}{e^{S_{k}}}>e^{\varepsilon \sigma \sqrt{n}}\right) \leqslant e^{-\varepsilon \sigma \sqrt{n}}
$$

то есть

$$
\mathbf{P}\left(\sup _{t \in[0, u]} \frac{\ln Y_{n t}}{\sigma \sqrt{n}}>\varepsilon, Z_{n}>0\right)=\mathbf{E}\left[\mathbb{P}\left(\sup _{t \in[0, u]} \frac{\ln Y_{n t}}{\sigma \sqrt{n}}>\varepsilon, Z_{n}>0\right)\right] \leqslant e^{-\varepsilon \sigma \sqrt{n}} \rightarrow 0
$$

при $n \rightarrow \infty$. Из (17), (81)-(83) следует (80). Теорема доказана.

\section{4. Некоторые важные следствия}

Далее мы будем предполагать, что выполняется следующее условие.

Условие Ао. Цепь Маркова $\left\{e_{n}\right\}$ удовлетворяет условию Деблина [5], является апериодичной и у нее существует только один эргодический класс.

Известно [5], что если выполнено условие А0, то цепь $\left\{e_{n}\right\}$ имеет стационарную меру $\pi:=\{\pi(i)\}_{i=0}^{\infty}$, причем $|p(n, i, j)-\pi(j)| \leqslant C \theta^{n}$, где $p(n, i, j):=$ $\mathbf{P}\left(e_{n}=j \mid e_{0}=i\right), C>0, \theta \in(0,1)$. Для $j \in \mathbf{N}_{0}$ будем снабжать верхним индексом $(j)$ обозначения случайных элементов, связанных с цепью $\left\{e_{n}\right\}$, если $e_{0}=j$ (например, $Z_{n}^{(j)}, S_{n}^{(j)}=X_{1}^{(j)}+\ldots+X_{n}^{(j)}$ и т.д.)

Заметим, что случайные величины $X_{i}, i \in \mathbf{N}$, и $\eta_{j}, j \in \mathbf{N}_{0},($ см. (4) и (5)) являются функциями от состояний $e_{i-1}$ и $e_{j}$ соответственно. Известно [5], что если

$$
\mathbf{E}^{(\pi)} \eta_{1}<\infty
$$


то с вероятностью 1

$$
\frac{1}{n} \sum_{i=0}^{n} \eta_{i}^{(j)} \rightarrow \mathbf{E}^{(\pi)} \eta_{1}
$$

равномерно по $j \in \mathbf{N}_{0}$. Предположим, что справедливы соотношения

$$
\mathbf{E}^{(\pi)} X_{1}=0
$$

и для некоторого $\delta_{0}>0$

$$
\mathbf{E}^{(\pi)}\left|X_{1}\right|^{2+\delta_{0}}<\infty .
$$

Известно также [5], что из (86) и (87) следует, что с вероятностью 1

$$
\frac{S_{n}^{(j)}}{n} \rightarrow 0
$$

равномерно по $j \in \mathbf{N}_{0}$ и что существует

$$
\lim _{n \rightarrow \infty} \mathbf{E}^{(\pi)}\left(n^{-1 / 2} S_{n}\right)^{2}=: \sigma_{1}^{2},
$$

причем если

$$
\sigma_{1}>0
$$

то (см. [4],[5]) равномерно по $j \in \mathbf{N}_{0}$

$$
\left\{\frac{S_{n t}^{(j)}}{\sigma_{1} \sqrt{n}}, t \in[0,1]\right\} \stackrel{\mathcal{D}}{\rightarrow}\{W(t), t \in[0,1]\},
$$

где $W(t)$ - стандартное броуновское движение.

В дальнейшем будем предполагать, что вместо условий А2 и А3 выполняются следующие условия $\mathrm{A} 2{ }^{*}$ и $\mathrm{A} 3^{*}$.

Условие А2*. Справедливы соотношения (84), (86), (87) и (88).

Условие $\mathbf{A 3}^{*}$. Существует $\beta>2$ такое, что $\lim _{x \rightarrow \infty} x^{\beta} g(x)=0$.

Утверждение 1. Если выполнены условия $A 0, A 1, A 2^{*}, A 3^{*}$ u $A 4$, то при $n \rightarrow \infty$

$$
\begin{gathered}
\left\{\frac{\ln \left(Z_{n t}+1\right)}{\sigma_{1} \sqrt{n}}, t \in[0, \infty) \mid Z_{n}>0\right\} \stackrel{\mathcal{D}}{\rightarrow}\left\{W^{+}(t \wedge \tau), t \in[0, \infty)\right\}, \\
\left\{\frac{\ln \left(Z_{n t}+1\right)}{\sigma_{1} \sqrt{n}}, \frac{t T}{n}, t \in[0, \infty) \mid Z_{n}>0\right\} \stackrel{\mathcal{D}}{\rightarrow}\left\{W^{+}(t \wedge \tau), t \tau, t \in[0, \infty)\right\}, \\
\left\{\frac{\ln \left(Z_{t T}+1\right)}{\sigma_{1} \sqrt{n}}, t \in[0,1] \mid Z_{n}>0\right\} \stackrel{\mathcal{D}}{\rightarrow}\left\{\frac{W^{+}(t)}{\alpha}, t \in[0,1]\right\},
\end{gathered}
$$

где $\tau$ - из (9), $\alpha$ - случайная величина, имеющая равномерное распределение на $(0,1)$ и не зависящая от $W^{+}$.

Прежде чем переходить к доказательству утверждения 1, установим следующую вспомогательную лемму.

Лемма 5. Пусть выполнены условия $A 0$ и $A 2^{*}$. Тогда при любом $\varepsilon>0$

$$
\lim _{\delta \downarrow 0} \lim _{n \rightarrow \infty} \frac{1}{\delta} \sup _{j \in \mathbf{N}_{0}} \mathbf{E}\left[\left(\mathbb{P}\left(Z_{n \delta}^{(j)}=0\right)\right)^{\exp (\varepsilon \delta \sqrt{n})}\right]=0 .
$$


Доказательство. Известно (формула (3.4) из [10]), что для $\delta \in(0,1)$

$$
\mathbb{P}\left(Z_{n \delta}^{(j)}=0\right) \leqslant 1-\frac{1}{a_{n \delta}^{(j)}+b_{n \delta}^{(j)}},
$$

где

$$
a_{n \delta}^{(j)}:=\exp \left\{-S_{n \delta}^{(j)}\right\}, b_{n \delta}^{(j)}:=\sum_{i=0}^{n \delta-1} \eta_{i}^{(j)} \exp \left\{-S_{i}^{(j)}\right\} .
$$

Далее надо, исходя из соотношений (89) и (85), практически дословно повторить доказательство леммы 4 из [9]. Лемма доказана.

Доказательство утверждения 1. Заметим сначала, что условия A0, A1, А2* влекут справедливость (2), причем

$$
\sigma_{1}=\sigma V^{-1 / 2}
$$

Несложно видеть, что

$$
\eta=\sum_{i=0}^{\nu-1} \frac{f_{i}^{\prime \prime}(1)}{\left(f_{i}^{\prime}(1)\right)^{2}} e^{-S_{i}}
$$

Значит, из условия А2* следует, что выполняется соотношение (3) с $q=\beta$. Таким образом, выполнены все условия теоремы 2.

Чтобы установить сходимость (90), достаточно доказать сходимость конечномерных распределений последовательности случайных процессов

$$
\mathcal{Y}_{n}=\mathcal{Y}_{n, t}:=\frac{\ln \left(Z_{n t}+1\right)}{\sigma_{1} \sqrt{n}}, t \in[0, \infty), n \in \mathbf{N}_{0},
$$

рассматриваемых при условии $Z_{n}>0$, и проверить, что при любом фиксированном $b>0$

$$
\lim _{\delta \downarrow 0} \overline{\lim }_{n \rightarrow \infty} \mathbf{P}\left(w_{\mathcal{Y}_{n}}(\delta, 0, b)>\varepsilon \mid Z_{n}>0\right)=0 .
$$

Зафиксируем $k \in \mathbf{N}$, неотрицательные $x_{i}, i=1, \ldots, k$, и $0 \leqslant t_{1}<t_{2}<\ldots<t_{k}$. Из теоремы 2 и равенства (94) следует, что при $t_{k} \leqslant 1$

$$
\lim _{n \rightarrow \infty} \mathbf{P}\left(\mathcal{Y}_{n, t_{i}}>x_{i}, i=1, \ldots, k \mid Z_{n}>0\right)=\mathbf{P}\left(W^{+}\left(t_{i} \wedge \tau\right)>x_{i}, i=1, \ldots, k\right),
$$

а при $b \leqslant 1$ выполняется соотношение (95). В общем случае проверка справедливости соотношений (96) и (95) практически совпадает с проверкой аналогичных утверждений при доказательстве теоремы 3 в [9] с той лишь разницей, что при упоминании о явном виде асимптотики вероятности невырождения процесса надо сослаться на лемму 2 (или на [18]), а также заметить, что для рассматриваемой нами марковской среды справедлив приводимый ниже аналог формулы (29) из [9], выведенной для случая среды, порожденной последовательностью независимых одинаково распределенных случайных величин. А именно, пусть $\delta \in(0,1)$,

$$
t_{1}:=1+(k-1) \delta, t_{2}:=t_{1}+2 \delta, \varepsilon>0, x(\varepsilon, n):=\exp \left(\varepsilon \sigma_{1} \sqrt{n}\right)-1,
$$

$T_{x(\varepsilon, n)}:=\inf \left\{n \geqslant n t_{1}: Z_{n} \in(x(\varepsilon, n), \infty)\right\}$ - первый момент достижения полуоси $(x(\varepsilon, n), \infty)$ процессом $\left\{Z_{n}, n \geqslant n t_{1}\right\}, \sum_{i, m, l}$ - знак суммирования по наборам $i \in \mathbf{N}_{0}$, $m \in\left[n t_{1}, n t_{2}\right], l \in(x(\varepsilon, n), \infty)$. Тогда

$$
\mathbf{P}\left(\exists n_{1}, n_{2} \in\left[n t_{1}, n t_{2}\right]: Z_{n_{1}}>\exp \left(\varepsilon \sigma_{1} \sqrt{n}\right)-1, Z_{n_{2}}=0\right)
$$




$$
\begin{aligned}
& =\mathbf{E} \sum_{i, m, l} \mathbb{P}\left(T_{x(\varepsilon, n)}=m, Z_{m}=l, e_{m}=i\right) \mathbb{P}\left(Z_{n t_{2}-m}^{(i)}=0\right)^{l} \\
& \leqslant \sum_{i, m, l} \mathbf{P}\left(T_{x(\varepsilon, n)}=m, Z_{m}=l, e_{m}=i\right) \mathbf{E}\left(Z_{3 \delta n}^{(i)}=0\right)^{\exp \left(\varepsilon \sigma_{1} \sqrt{n}\right)} \\
& \leqslant \sup _{i \in \mathbf{N}_{0}} \mathbf{E}\left(Z_{3 \delta n}^{(i)}=0\right)^{\exp \left(\varepsilon \sigma_{1} \sqrt{n}\right)} \mathbf{P}\left(T>n t_{1}\right) .
\end{aligned}
$$

Известно (см. доказательство теорем $3,4,5$ в [9]), что из соотношений (93) и (97) следует сходимость (90), которая, в свою очередь, влечет справедливость соотношений (91) и (92).

Утверждение 2. Если выполнены условия $A 0, A 1, A 2^{*}, A 3^{*}$ u $A 4$, то при $x \rightarrow \infty$

$$
\mathbf{P}\left(\sup _{n \in \mathbf{N}_{0}} Z_{n}>x\right) \sim \frac{c}{\ln x},
$$

где $c>0$.

Доказательство. Пусть $\varepsilon>0, x>0, d:=\exp x-1, T_{d}:=\inf \left\{n \geqslant 0: Z_{n} \in(d, \infty)\right\}$, $\sum_{i, k, l}$ - знак суммирования по наборам $i \in \mathbf{N}_{0}, k \in\left[0, \varepsilon x^{2}\right], l \in(d, \infty)$. Справедливость (98) выводится из соотношений (17), (92) и (93) с помощью практически тех же рассуждений, которые были проведены при доказательстве теоремы 1 в [9], при этом нужно учесть, что ввиду (??) (аналогично (97)) выполняется соотношение

$$
\begin{gathered}
\mathbf{P}\left(\sup _{n \in \mathbf{N}_{0}} Z_{n}>d, T \leqslant \varepsilon x^{2}\right) \\
=\mathbf{E} \sum_{i, k, l} \mathbb{P}\left(T_{d}=k, Z_{k}=l, e_{k}=i\right) \mathbb{P}\left(Z_{\varepsilon x^{2}-k}^{(i)}=0\right)^{l} \\
=\sum_{i, k, l} \mathbf{P}\left(T_{d}=k, Z_{k}=l, e_{k}=i\right) \mathbf{E}\left[\mathbb{P}\left(Z_{\varepsilon x^{2}-k}^{(i)}=0\right)^{l}\right] \\
\leqslant \sup _{i \in \mathbf{N}_{0}} \mathbf{E}\left(1-\frac{1}{a_{\varepsilon x^{2}}^{(i)}+b_{\varepsilon x^{2}}^{(i)}}\right)^{\exp x-1} \mathbf{P}\left(\sup _{n \in \mathbf{N}_{0}} Z_{n}>d\right),
\end{gathered}
$$

и применить лемму 5.

Следствие 1. Если выполнены условия $A 0, A 1, A 2^{*}, A 3^{*}$ и $A 4$, то при $x \rightarrow \infty$

$$
\mathbf{P}\left(\sum_{n=0}^{\infty} Z_{n}>x\right) \sim \frac{c}{\ln x},
$$

где с >0 то же, что и в утверждении 2.

Доказательство. Известно (см. доказательство теоремы 2 в [9]), что соотношения (17), (98) влекут (99).

Автор выражает благодарность рецензенту, замечания которого способствовали улучшению работы. 


\section{Список литературы}

1. Smith W.L., Wilkinson W., "On branching processes in random environment", Ann. Math. Statist., 40:3 (1969), 814-827.

2. Athreya K. B., Karlin S., "On branching processes with random environments: I,II.", Ann. Math. Statist., 42:5, 6 (1971), 1499-1520, 1843-1858.

3. Биллингли П., "Сходимость вероятностных мер", 1977.

4. Billinsley P., "The invariance principle for dependent random variables", Trans. Amer. Math. Soc., 83:1 (1956), 250-268.

5. Дуб Дж. Л., Вероятностные прочессы, ИЛ, Москва, 1956.

6. Козлов М.В., "Об асимптотике вероятности невырождения критических ветвящихся процессов в случайной среде", Теория вероятн. и ее применен., 21:4 (1976), 813-825.

7. Афанасьев В.И., "Предельная теорема для критического ветвящегося процесса в случайной среде", Дискретная математика, 5:1 (1993), 45-58.

8. Афанасьев В.И., "Новая предельная теорема для критического ветвящегося процесса в случайной среде", Дискретная математика, 9:3 (1997), 52-67.

9. Афанасьев В.И., "О максимуме критического ветвящегося процесса в случайной среде”, Дискретная математика, 11:2 (1999), 86-102.

10. Afanasyev V.I., Geiger J., Kersting G., Vatutin V.A., "Criticality for branching processes in random environment", Ann. Prob., 33:2 (2005), 645-673.

11. Afanasyev V.I., Böinghoff K., Kersting G., Vatutin V.A., "Limit theorems for weakly subcritical branching processes in random environment", J. Theoret. Probab., 25:3 (2012), 703-732.

12. Geiger J., Kersting G., "The survival probability of a critical branching process in random environment", Теория вероятн. и ее применен., 45:3 (2000), 607-615.

13. Боровков А.А., Теория вероятностей, М.: Наука, 1999.

14. Ватутин В.А., Дьяконова Е.Е., "Ветвящиеся процессы Гальтона-Ватсона в случайной среде, I: предельные теоремы", Теория вероятн. и ее применен., 48:2 (2003), 274-300.

15. Ватутин В.А., Дьяконова Е.Е., "Ветвящиеся процессы Гальтона-Ватсона в случайной среде, II: конечномерные распределения", Теория вероятн. и ее применен., 49:2 (2004), 231-268.

16. Ватутин В.А., Дьяконова Е.Е., “Ветвящиеся процессы в случайной среде и бутылочные горлышки в эволюции популяций”, Теория вероятн. и ее применен., 51:1 (2006), 22-46.

17. Ватутин В.А., Вахтель В.И., "Внезапное вырождение критического ветвящегося процесса в случайной среде", Теория вероятн. и ее применен., 54:3 (2009), 417-438.

18. Дьяконова Е.Е., "Многотипные ветвящиеся процессы Гальтона-Ватсона в марковской случайной среде", Теория вероятн. и ее применен., 55:3 (2011), 592-601.

19. Дьяконова Е.Е., "Многотипные ветвящиеся процессы, эволюционирующие в марковской случайной среде", Дискретная математика, 24:3 (2012), 130-151.

20. Dyakonova E.E., Geiger J., Vatutin V.A., "On the survival probability and a functional limit theorem for branching processes in random environment", Markov Process. Related Fields, 10:2 (2004), 289-306.

21. Le Page E., Ye Y., "The survival probability of a critical branching process in a Markovian random environment", C.R. Acad. Sci. Paris, Ser. I, 348, 2010, 301-304.

22. D' Souza J.C., Hambly B.M., "On the survival probability of a branching process in a random environment", Adv. Appl. Prob., 29:1 (1997), 38-55. 